\title{
Predictors of chronic kidney disease in obstructive sleep apnea patients
}

\author{
Phunphai Somkearti, Paiboon Chattakul, Sittichai Khamsai, Panita Limpawattana, Jarin Chindaprasirt, \\ Verajit Chotmongkol, Kittisak Sawanyawisuth
}

Department of Medicine, Faculty of Medicine, Sleep Apnea Research Group, Research Center in Back, Neck and Other Joint Pain and Human Performance, Khon Kaen University, Khon Kaen, Thailand

Introduction: Obstructive sleep apnea (OSA) is a common condition in patients with chronic kidney disease (CKD). It may worsen renal function in CKD patients and is associated with uncontrolled blood pressure. Although OSA is found in up to $80 \%$ of CKD patients, there are limited data available on its clinical features in patients with and without CKD.

Objective: This study aimed to identifying the differences in the clinical characteristics of OSA between CKD and non-CKD OSA patients and determine the clinical predictors for CKD in OSA patients.

Methods: This was a retrospective study conducted at Khon Kaen University's Srinagarind Hospital in Thailand between July and December 2018. The inclusion criteria were diagnosis with OSA via polysomnography and having undergone laboratory tests for CKD. Obstructive sleep apnea is diagnosed according to the apnea-hypopnea index (AHI) as experiencing $\geq 5$ events/hour, while CKD diagnosed based on the KDOQI guidelines. Eligible patients were divided into two groups: OSA with CKD and OSA without CKD. Predictors of CKD in OSA patients were analyzed using multivariate logistic regression analysis.

Results: During the study period, there were 178 OSA patients who met the study criteria, $88(49.44 \%)$ of whom were in the OSA with CKD group. Both age and body mass index were comparable between OSA patients with CKD and those without (age: 59 and 57 years, respectively; body mass index: 30 and $29 \mathrm{~kg} / \mathrm{m}^{2}$, respectively. There were three significant factors that differed between those with and without CKD group including systolic blood pressure (147 vs $135 \mathrm{mmHg}$ ), proportion of patients with diabetes (55\% vs 34\%), and proportion of patients with Mallampati scores of 3-4 (73\% vs 39\%). There were three independent predictors for OSA in patients with CKD: female sex, high systolic blood pressure, and Mallampati score of 3 or 4, with adjusted odds ratios (95\% confidence interval) of 4.624 (1.554, 13.750), $1.060(1.020,1.101)$, and $2.816(1.356,5.849)$, respectively. The Hosmer-Lemeshow chi-square statistic of the predictive model was 6.06 (p 0.640). Systolic blood pressure of more than 130 and $150 \mathrm{mmHg}$ resulted in sensitivity of $84.21 \%$ and specificity of $81.40 \%$, respectively.

Conclusions: Female sex, high systolic blood pressure, and Mallampati score of 3-4 were suggestive of OSA with CKD. Obstructive sleep apnea patients with one or more of these predictors may have a high risk of CKD.

Key words: Predictors, systolic blood pressure, sex.

Correspondence: Kittisak Sawanyawisuth, MD, PhD, Department of Medicine, Faculty of Medicine, Khon Kaen University, Khon Kaen, 40002, Thailand. Tel: +66.43.363664 - Fax: +66.43.348399. E-mail: kittisak@kku.ac.th

Contributions: PS, KS, study concept and design, manuscript drafting; KS, statistical analyses performing; PC, SK, PL, JC, $\mathrm{VC}$, data interpretation, contribution to discussion. All authors reviewed the manuscript, read, and approved the final manuscript.

Conflict of interest: The Authors declare no conflict of interest.

Funding: None.

Availability of data and materials: Data are available upon request.

Ethics approval and consent to participate: All procedures performed in studies involving human participants were in accordance with the ethical standards of the institutional and/or national research committee and with the 1964 Helsinki declaration and its later amendments or comparable ethical standards. The study was reviewed and approved by the Khon Kaen University Ethics Committee of Human Research (no. HE611131).

Informed consent: Informed consent was waived due to retrospective study design according to the law of Thailand.

Consent for publication: Not applicable. 


\section{Introduction}

Obstructive sleep apnea (OSA) is categorized by cyclic obstruction of the upper airway during sleep. It has various clinical manifestations, from loud snoring, excessive daytime somnolence, and apneas. The condition is diagnosed via polysomnography according to the apnea-hypopnea index (AHI) as experiencing $\geq 5$ events/hour [1]. Obstructive sleep apnea can exacerbate various other conditions such as hypertension, heart failure, stroke, gastroesophageal reflux disease, and chronic kidney disease (CKD) [2]. There is evidence of a bidirectional relationship between OSA and CKD. Chronic kidney disease may potentiate sleep apnea due to fluid redistribution and unstable chemosensitivity of the respiratory control system, while hypoxemia and sympathetic surges associated with arousals and systemic inflammation in OSA may lead to kidney injury [3]. Obstructive sleep apnea is found in nearly $80 \%$ in CKD patients [4] and some studies have suggested that OSA is a risk factor for CKD [5-7]. A study from Japan, for example, found that $30.5 \%$ of OSA patients had CKD, a rate three times greater than that in the general population $(9.1 \%)$ [8]. It is difficult to draw the conclusion that worsening kidney function in these patients is solely a result of OSA as they usually have other comorbidities including diabetes mellitus, obesity, and hypertension. However, a cohort study from Taiwan showed that even those OSA patients without hypertension or diabetes were at higher risk of developing CKD and experienced earlier onset of CKD compared to a control group [9]. Although OSA and CKD are related, there are limited data available regarding the factors that predict CKD in OSA patients. This study thus aimed to determine the clinical predictors for CKD in those patients.

\section{Methods}

We conducted a retrospective, cross-sectional, analytic study at Khon Kaen University's Srinagarind Hospital in Thailand. The study period was between July and December 2018. The inclusion criteria were i) age $\geq 18$ years; ii) having been diagnosed with OSA via polysomnography; and iii) having undergone laboratory tests for CKD. Obstructive sleep apnea is diagnosed according to the apnea-hypopnea index (AHI) as experiencing $\geq 5$ events/hour, while CKD is diagnosed based on the KDOQI guidelines. The polysomnography used in this study was Alice $\mathrm{PDX}^{\circledR}$ as a home sleep study (Type 3 polysomnography) which had agreement of $96.4 \%$ with reference polysomnography. The scoring criteria for apnea and hypopnea are as follows: apnea: at least a 90\% reduction of airflow for at least 10 seconds with at least $90 \%$ of the event's duration meets the amplitude reduction criteria for apnea, hypopnea: either $\geq 30 \%$ reduction in airflow for at least 10 s with $\geq 4 \%$ desaturation or $\geq 50 \%$ reduction in airflow for at least 10 s with $\geq 3 \%$ desaturation [10].

Baseline characteristics, comorbidities, OSA symptoms/ signs/complications, and laboratory results of all eligible patients were examined and recorded. All data were recorded at the time of OSA diagnosis. Excessive daytime sleepiness or other OSA symptoms were evaluated by subjective evaluation or self-reported questionnaire by using the STOP Bang questionnaire.

Sample size calculation: the prevalence of CKD in cases of OSA is $30 \%$. Based on a $95 \%$ confidence interval, the required sample size was determined to be 82 according to the infinite population proportion described by Ngamjarus et al. [11].

Statistical analysis: baseline and clinical characteristics of OSA patients with and without CKD were compared using descriptive statistics. When appropriate, a Wilcoxon rank sum/Student's $t$-test and Fisher's exact tests/chi-square test were applied to compare the differences between the two groups in terms of numbers and proportions, respectively. Univariate logistic regression analysis was applied to calculate the crude odds ratio (OR) of individual variables for OSA associated with CKD. Those factors with a $p$ of less than 0.20 by univariate logistic regression analysis and clinically significant variables were included in subsequent multivariate logistic regression analysis. Analytical results were presented as crude OR, adjusted OR, and 95\% confidence intervals. The goodness of fit of the multivariate logistic regression model was tested using the Hosmer-Lemeshow method. Numerical independent factors for OSA associated with CKD were analyzed for appropriate cutoff points. Sensitivities and specificities for various cutoff points for CKD were also computed. All data analysis was performed using STATA software (StataCorp LP, College Station, TX, USA). The study was reviewed and approved by the Khon Kaen University Ethics Committee of Human Research (no. HE611131).

\section{Results}

There were 178 OSA patients included into the study, 88 (49.44\%) of whom had CKD. The baseline characteristics, physical signs, and laboratory results of OSA patients with and without CKD are shown in Tables 1-3. For the CKD group, were 45 patients $(51.13 \%)$ with identified causes of CKD as follows: 31 patients with albuminuria, 10 patients with proteinuria $>1 \mathrm{gm}, 2$ patients with obstructive uropathy, 1 patient with IgA nephropathy, and 1 patient with focal segmental glomerulosclerosis (FSGS). Additionally, 10 patients were on dialysis.

Baseline characteristics of the two groups were comparable. For example, median age was 59 vs 57, and body mass index was 30 vs $29 \mathrm{~kg} / \mathrm{m}^{2}$ in the CKD and non-CKD group, respectively. However, the proportion of patients with hypertension and dia-

Table 1. Baseline characteristic of obstructive sleep apnea patients with and without chronic kidney disease.

\begin{tabular}{lccc} 
Factor & $\begin{array}{c}\text { CKD } \\
(\mathbf{n = 8 8})\end{array}$ & $\begin{array}{c}\text { Non-CKD } \\
(\mathbf{n = 9 0})\end{array}$ & $\boldsymbol{p}$ \\
Male sex & $51(57.95)$ & $56(62.2)$ & 0.646 \\
Median (1 ${ }^{\text {st-3rd }}$ quartile) age, years & $59(44-66)$ & $57(47-66)$ & 0.831 \\
\hline Excessive daytime sleepiness & $39(68)$ & $39(58.21)$ & 0.336 \\
Tiredness or fatigue & $14(70.00)$ & $38(80.85)$ & 0.352 \\
\hline STOP-BANG questionnaire score & $5(4-5)$ & $5(4-6)$ & 0.780 \\
Diabetes mellitus & $47(55.29)$ & $27(34.62)$ & 0.012 \\
\hline Coronary artery disease & $6(7.06)$ & $0(0.0)$ & 0.029 \\
Heart failure & $12(14.12)$ & $9(11.54)$ & 0.648 \\
\hline Atrial fibrillation & $15(17.05)$ & $16(17.78)$ & 0.995 \\
Hypertension & $81(95.29)$ & $58(74.36)$ & $<0.001$ \\
\hline Stroke & $7(8.24)$ & $6(7.69)$ & 0.99 \\
Gastroesophageal reflux disease & $14(16.47)$ & $12(15.38)$ & 0.99 \\
\hline Gout & $13(15.29)$ & $5(6.41)$ & 0.083 \\
Allergic rhinitis & $22(25.88)$ & $13(16.67)$ & 0.183 \\
\hline Previous smoking & $10(25.00)$ & $12(20.69)$ & 0.631 \\
Previous alcohol consumption & $11(28.95)$ & $9(16.07)$ & 0.199 \\
\hline Current smoking & $1(2.50)$ & $5(8.93)$ & 0.396 \\
Current alcohol consumption & $1(3.33)$ & $4(7.02)$ & 0.656 \\
\hline
\end{tabular}

CKD, chronic kidney disease. Data are presented as number (percentage) unless indicated otherwise. 
betes mellitus differed significantly between the two groups $(81 \%$ vs $58 \%$ for hypertension with a $p$ of $<0.001$ and $55.29 \%$ vs $34.62 \%$ for diabetes mellitus with a $p$ of 0.012 ). As shown in Table 2, OSA patients with CKD had significantly higher systolic blood pressure than those without (147 vs $135 \mathrm{mmHg}$; $\mathrm{p}<0.001)$. The proportions of patients with Mallampati scores of 3-4 were also significantly different between the two groups $(73 \%$ vs $39 \%$; $<<0.001)$. In addition, apnea-hypopnea index (AHI) scores were significantly higher in the CKD group than in the non-CKD group (23.5 vs 14 events/h; $\mathrm{p}=0.006$ ), as shown in Table 3 . Other laboratory results in which there were significant differences between the two groups include UACR, uric acid, and neutrophil and lymphocyte percentages (Table 3).

There were three independent predictors for CKD in OSA patients according to multivariate regression analysis: sex, systolic blood pressure, and Mallampati classification (Table 4), with odds ratios (95\% confidence interval) of 4.624 (1.554, 13.757), 1.060 $(1.020,1.101)$, and $2.816(1.356,5.849)$, respectively. The Hosmer-Lemeshow chi-square of the predictive model was 6.06 $(\mathrm{p}=0.640)$. Systolic blood pressure of 130 and $150 \mathrm{mmHg}$ cut points resulted in sensitivity of $84.21 \%$ and specificity of $81.40 \%$, respectively.

\section{Discussion}

We found that female OSA patients tended to have a five-times higher risk of CKD than their male counterparts. Sex is an important factor that affects prevalence, clinical features, and clinical outcomes [12]. However, the reason for this correlation has yet to be explained. One possible explanation is that OSA symptoms in female patients tend to be less severe, leading these patients to seek medical treatment later and resulting in greater CKD development [13]. Another reason may have to do with endothelial dysfunction

Table 2. Physical signs of obstructive sleep apnea patients with and without chronic kidney disease.

\begin{tabular}{|c|c|c|c|}
\hline Factor & $\begin{array}{c}\text { CKD } \\
(\mathrm{n}=88)\end{array}$ & $\begin{array}{c}\text { Non-CKD } \\
(n=90)\end{array}$ & $p$ \\
\hline Body mass index, $\mathrm{kg} / \mathrm{m}^{2}$ & $30.4(26.27-35)$ & $29(25.4-33.1)$ & 0.248 \\
\hline Neck circumference, $\mathrm{cm}$ & $41(39-45)$ & $41(39-44)$ & 0.911 \\
\hline Median (1 $1^{\text {st }-3 \text { rd }}$ quartile) SBP, mmHg & $146.5(134-156)$ & $135(123-146)$ & $<0.001$ \\
\hline Median (1 $1^{\text {st- }-3 \text { rd } q u a r t i l e) ~ D B P, ~} \mathrm{mmHg}$ & $80(70-86)$ & $79(72-85)$ & 0.929 \\
\hline Median (1 $1^{\text {st-3rd }}$ quartile) BMI, $\mathrm{kg} / \mathrm{m}^{2}$ & $30.4(26.27-35)$ & $29(25.4-33.1)$ & 0.248 \\
\hline $\begin{array}{l}\text { Median (1 } 1^{\text {st }} 3^{\text {rd }} \text { quartile) } \\
\text { neck circumference, cm }\end{array}$ & $41(39.0-45.0)$ & $41(39.0-44.0)$ & 0.911 \\
\hline $\begin{array}{l}\text { Mallampati classification } \\
\text { Class I } \\
\text { Class II } \\
\text { Class III } \\
\text { Class IV }\end{array}$ & $\begin{array}{c}2(3.85) \\
12(23.08) \\
24(46.15) \\
14(26.92)\end{array}$ & $\begin{array}{c}10(16.39) \\
27(44.26) \\
21(34.43) \\
3(4.92)\end{array}$ & $<0.001$ \\
\hline Macroglossia & $23(34.85)$ & $23(31.08)$ & 0.719 \\
\hline Torus palatinus & $1(1.56)$ & $8(11.11)$ & 0.036 \\
\hline Torus mandibularis & $2(3.17)$ & $4(5.71)$ & 0.683 \\
\hline Tonsillar enlargement & $28(31.82)$ & $36(40.00)$ & 0.277 \\
\hline Microretrognathia & $2(3.13)$ & $2(2.74)$ & 0.990 \\
\hline Retrognathia & $3(4.92)$ & $5(7.04)$ & 0.725 \\
\hline
\end{tabular}

CKD, chronic kidney disease; SBP, systolic blood pressure; DBP, diastolic blood pressure; BMI, body mass index. Data are presented as number (percentage) unless indicated otherwise. and inflammation [14-16]. Hypoxemia may stimulate the release of cytokine and free radical products in OSA patients, which leads to a reductions in nitric oxide. Previous studies have found that high levels of various inflammatory proteins are significantly correlated with OSA severity. Surprisingly, increases in C-reactive protein (CRP) levels and the Erythrocyte Sedimentation Rate (ESR) in female OSA patients were found to be greater than those in their male counterparts [17]. Endothelial function was also found to be more sensitive and impaired in female OSA patients than in males. The mechanisms behind this difference have yet to

Table 3. Laboratory investigation results of obstructive sleep apnea (OSA) patients with and without chronic kidney disease.

\begin{tabular}{|c|c|c|c|}
\hline Factor & $\begin{array}{c}\text { CKD } \\
(n=88)\end{array}$ & $\begin{array}{c}\text { Non-CKD } \\
(n=90)\end{array}$ & $p$ \\
\hline Blood urea nitrogen, mg/dL & 21.1(13.7-38.3) & 13.3(11.5-15.7) & $<0.001$ \\
\hline Serum creatinine, mg/dL & $1.4(1.1-2.45)$ & $0.9(0.76-1.1)$ & $<0.001$ \\
\hline GFR & $47.5(28-66.5)$ & $85.5(73-99.7)$ & $<0.001$ \\
\hline Uric, mg/dL & $7.2(5.9-8.3)$ & $6.1(5.2-7)$ & 0.002 \\
\hline UACR, mg/g & $93.5(29.2-560.2)$ & $4.64(2.4-12.01)$ & $<0.001$ \\
\hline $\mathrm{HbAlC}, \%$ & $6.6(5.65-8.25)$ & $6.1(5.5-7)$ & 0.060 \\
\hline Cholesterol, mg/dL & $179(152-209)$ & $195(170-220)$ & 0.149 \\
\hline Triglyceride, mg/dL & 147(87-188) & $148(97-202)$ & 0.448 \\
\hline HDL-c, mg/dL, mg/dL & $47(39-56)$ & $48(42-59)$ & 0.149 \\
\hline LDL-c, mg/dL, mg/dL & $117(93-159)$ & $130(96-158)$ & 0.288 \\
\hline Neutrophil, \% & $61.8(53.6-65.6)$ & $53.8(48.4-60.8)$ & 0.002 \\
\hline Lymphocyte, \% & $26.7(20.4-30.8)$ & $30.9(27-37.2)$ & 0.001 \\
\hline Platelet, mm³ & $242 \times 10^{3}(203-282)$ & $233 \times 10^{3}(188-282)$ & 0.311 \\
\hline LVEF, \% & $66(58-70)$ & $67(62-75)$ & 0.475 \\
\hline RVSP, mmHg & 29.59(19.9-51.73) & 28.66(21.1-46.97) & 0.899 \\
\hline AHI, per hour & 23.5(14.7-47) & $14(9-30)$ & 0.006 \\
\hline $\begin{array}{l}\text { Severity of OSA, \% } \\
\text { Mild } \\
\text { Moderate } \\
\text { Severe }\end{array}$ & $\begin{array}{l}25 \% \\
30 \% \\
45 \%\end{array}$ & $\begin{array}{l}0.006 \\
52 \% \\
21 \% \\
27 \%\end{array}$ & \\
\hline Lowest $\mathrm{O}_{2}, \%$ & $81(72-88.5)$ & $85(73-90)$ & 0.338 \\
\hline
\end{tabular}

CKD, chronic kidney disease; HDL-c, high density lipoprotein-cholesterol; LDL-c, low density lipoprotein-cholesterol; UCAR, urine albumin creatinine ratio; LVEF, Left ventricular ejection fraction; RVSP, right ventricular systolic pressure; $\mathrm{AHI}$, apnea hypopnea index. Data presented as median ( $1^{\text {st- }-3 \text { rd }}$ percentile) unless indicated otherwise.

Table 4. Factors associated with chronic kidney disease in obstructive sleep apnea patients according to logistic regression analysis.

\begin{tabular}{lll} 
Factors & $\begin{array}{l}\text { Unadjusted odds } \\
\text { ratio (95\% } \\
\text { confidence } \\
\text { interval) }\end{array}$ & $\begin{array}{l}\text { Adjusted odds } \\
\text { ratio }(95 \% \\
\text { confidence } \\
\text { interval) }\end{array}$ \\
\hline Female sex* & $1.195(0.655,4.624)$ & $4.624(1.554,13.750)$ \\
SBP, mmHg* & $1.039(1.019,1.060)$ & $1.060(1.020,1.101)$ \\
\hline Mallampati classification* & $2.829(1.676,4.776)$ & $2.816(1.356,5.849)$ \\
Diabetes mellitus & $2.336(1.240,4.398)$ & $0.934(0.312,2.792)$ \\
\hline Body mass index, $\mathrm{kg} / \mathrm{m}^{2}$ & $1.028(0.981,1.076)$ & $0.994(0.911,1.086)$ \\
\hline
\end{tabular}

*Independent predictor for chronic kidney disease in obstructive sleep apnea; SBP, systolic blood pressure. 
be determined, but menopause may be a factor, as estrogen can promote the production of nitric oxide and slow its degradation [18]. Although the mean age of our patients was over 50 years, we were not able to assess their menopause status. The relationship among OSA, hypertension, and CKD is well-documented [19, 20]. Hypoxia due to OSA leads to increases in sympathetic tone and vascular resistance through the activation of the renin angiotensin aldosterone system (RAAS), which is associated with the fibrosis of kidney [20]. Systolic blood pressure was an important predictor for renal disease progression in this study, which is consistent with the findings of several previous studies [21,22].

Mallampati score (the estimation of tongue size relative to the oral cavity) was also an independent factor for CKD in this study. A score of 3-4 increased the risk of CKD by nearly 3 times. To our knowledge, this is the first study to identify a high Mallampati score as being a risk factor for CKD development in OSA patients. We suspect that the fluid retention process might play role in increasing the tongue size, a phenomenon that has previously been shown in pregnant women [23]. A large tongue may lower oxygen levels during sleep, leading to a higher chance of developing CKD [24]. However, Mallampati scores are subjective and may vary from examination to examination.

We found that if OSA patients had systolic blood pressure over $130 \mathrm{mmHg}$, sensitivity of having CKD was $84.21 \%$. If the systolic blood pressure was over $150 \mathrm{mmHg}$, the specificity was $81.40 \%$. These data may imply two points as follows: i) OSA patients with high systolic blood pressure are at risk for CKD. Therefore, the physicians should be aware of CKD in OSA patients with systolic blood pressure over $130 \mathrm{mmHg}$. ii) OSA patients may need to have a target systolic blood pressure of $130 \mathrm{mmHg}$ to prevent risk of CKD. If the systolic blood pressure was over $150 \mathrm{mmHg}$, the specificity of having CKD in OSA patients would rise. In other words, the target blood pressure for OSA with hypertension may be appropriate at $130 \mathrm{mmHg}$. As the target systolic blood pressure has been set for other populations but not in OSA patients [25].

There were some limitations to this study. First, it was conducted in a single-site, referral medical center. Second, the predictive model for CKD included only clinical symptoms and signs and not laboratory results. The benefit of this model, however, is that it can be employed as a simple clinical model for health care facilities with limited resources. Other predictive models that include laboratory results should be developed. No intervention or evaluation of treatment outcomes were studied [26-28]. Due to retrospective data collection, some factors may be missing or not studied such as fluid distribution or menopausal status. Finally, SBP in this study was office blood pressure, and did not take into account home or nighttime blood pressure patterns. Due to the fact that OSA and CKD patients usually have non-dipping blood pressure variations [29], morning blood pressure or home-monitoring blood pressure should be examined further.

\section{Conclusion}

Female sex, high systolic blood pressure, and Mallampati classes 3-4 were suggestive of CKD in OSA patients.

\section{Acknowledgements}

The authors would like to thank Dr. Dylan Southard (USA) for his kind review of the final manuscript and Sleep Apnea Research Group, Khon Kaen University, Thailand.

\section{References}

1. Reutrakul S, Mokhlesi B. Obstructive sleep apnea and diabetes: A state of the art review. Chest 2017;152:1070-86.

2. Adeseun GA, Rosas SE. The impact of obstructive sleep apnea on chronic kidney disease. Curr Hypertens Rep 2010;12:378-83.

3. Abuyassin B, Sharma K, Ayas NT, Laher I. Obstructive sleep apnea and kidney disease: A potential bidirectional relationship. J Clin Sleep Med 2015;11:915-24.

4. Yayan J, Rasche K, Vlachou A. Obstructive sleep apnea and chronic kidney disease. Adv Exp Med Biol 2017;1022:11-8.

5. Kanbay A, Buyukoglan H, Ozdogan N, Kaya E, Oymak FS, Gulmez I, et al. Obstructive sleep apnea syndrome is related in the progression of chronic kidney disease. Int Urol Nephrol 2012;44:535-9.

6. García-Canton C, Bosch E, Ramírez A, Gonzalez Y, Auyanet I, Guerra R, et al. Obstructive sleep apnoea: a stand-alone risk factor for chronic kidney disease. Nephrol Dial Transplant 2011; 26:2250-6.

7. Hwu DW, Lin KD, Lin KC, Lee YJ, Chang YH. The association of obstructive sleep apnea and renal outcomes-a systematic review and meta-analysis. BMC Nephrol 2017;18:313.

8. Iseki K, Tohyama K, Matsumoto T, Nakamura H. High Prevalence of chronic kidney disease among patients with sleep related breathing disorder (SRBD). Hypertension Res 2008;31:249-55.

9. Lin YS, Liu PH, Lin SW, Chuang LP, Ho WJ, Chou YT, et al. Simple obstructive sleep apnea patients without hypertension or diabetes accelerate kidney dysfunction: a population followup cohort study from Taiwan. Sleep Breath 2017;21:85-91.

10. Nilius G, Domanski U, Schroeder M, Franke KJ, Hogrebe A, Margarit L, et al. A randomized controlled trial to validate the Alice PDX ambulatory device. Nat Sci Sleep 2017;9:171-80.

11. Ngamjarus C, Chongsuvivatwong V, McNell E. n4Studies: Sample size calculation for an epidemiological study on a smart device. Siriraj Med J 2016 68:160-70.

12. Basoglu OK, Tasbakan MS. Gender differences in clinical and polysomnographic features of obstructive sleep apnea: a clinical study of 2827 patients. Sleep Breath 2018;22:241-9.

13. Lastra AC, Attarian HP. The persistent gender bias in the diagnosis of obstructive sleep apnea: A commentary. Gender Genome 2018;2:43-8.

14. Seliger SL, Salimi S, Pierre V, Giffuni J, Katzel L, Parsa A. Microvascular endothelial dysfunction is associated with albuminuria and CKD in older adults. BMC Nephrol 2016;17:82.

15. Guerrrot D, Dussaule JC, Kavvadas P, Boffa JJ, Chadjichristos $\mathrm{CE}$, Chatziantoniou C. Progression of renal fibrosis: the underestimated role of endothelial alterations. Fibrogenesis Tissue Repair 2012;5:S15.

16. Johnson RJ, Nangaku M. Endothelial dysfunction: The secret agent driving kidney disease. J Am Soc Nephrol 2016;27:3-5.

17. Bouloukaki I, Mermigkis C, Tzanakis N, Kallergis E, Moniaki $\mathrm{V}$, Mauroudi E, et al. Evaluation of inflammatory markers in a large sample of obstructive sleep apnea patients without comorbidities. Mediators Inflamm 2017;2017:4573756.

18. Faulx MD, Larkin EK, Hoit BD, Aylor JE, Wright AT, Redline $\mathrm{S}$. Sex influences endothelial function in sleep-disordered breathing. Sleep 2004;27:1113-20.

19. Chang CP, Li TC, Hang LW, Liang SJ, Lin JJ, Chou CY, et al. The relationships of sleep apnea, hypertension, and resistant hypertension on chronic kidney disease. Medicine (Baltimore) 2016;95:e3859.

20. Aziz F, Chaudhary K. The triad of sleep apnea, hypertension, and chronic kidney disease: A spectrum of common pathology. Cardiorenal Med 2016;7:74-82. 
21. Mentari E, Rahman M. Blood pressure and progression of chronic kidney disease: importance of systolic, diastolic, or diurnal variation. Curr Hypertens Rep 2004;6:400-4.

22. Agarwal R, Andersen MJ. Correlates of systolic hypertension in patients with chronic kidney disease. Hypertension 2005;46:514-20.

23. Isono S. Mallampati classification, an estimate of upper airway anatomical balance, can change rapidly during labor. Anesthesiology 2008;108:347-9.

24. Ahn SH, Kim J, Min HJ, Chung HJ, Hong JM, Lee JG, et al. Tongue volume influences lowest oxygen saturation but not apnea-hypopnea index in obstructive sleep apnea. PLoS One 2015;10:e0135796.

25. Bakris G, Ali W, Parati G. ACC/AHA versus ESC/ESH on hypertension guidelines: JACC Guideline Comparison. J Am
Coll Cardiol 2019;73:3018-26.

26. Phitsanuwong C, Senthong V. CPAP therapy in a young hypertension patient. Asia Pac J Sci Technol 2016;21:APST-21-0401.

27. Phitsanuwong C, Ariyanuchitkul S, Chumjan S, Domthong A, Silaruks S, Senthong S. Does hypertensive crisis worsen the quality of life of hypertensive patients with OSA?: A pilot study. Asia Pac J Sci Technol 2017;22:APST-22-02-01.

28. Sawunyavisuth B. What are predictors for a continuous positive airway pressure machine purchasing in obstructive sleep apnea patients? Asia Pac J Sci Technol 2018;23:APST-23-0310.

29. Kario K. Nocturnal hypertension new technology and evidence. Hypertension 2018;71:997-1009. 